\title{
Color Image Enhancement Using Optimal Linear Transform with Hue Preserving and Saturation Scaling
}

\author{
Xiaohua Zhang ${ }^{1 *}$, Yuelan Xin ${ }^{2}$, Heming Huang ${ }^{2}$, Ning Xie ${ }^{3}$ \\ ${ }^{1}$ Hiroshima Institute of Technology, Hiroshima, 731-5193, Japan \\ ${ }^{2}$ Qinghai Normal University, Xining, 810008, China \\ ${ }^{3}$ Tongji University, Shanghai, 200092, China \\ * Corresponding author’s Email: zhxh@cc.it-hiroshima.ac.jp
}

\begin{abstract}
Although image contrast enhancement is a low level image processing issue, it is very important for improving image quality. This paper propose a new algorithm using optimal linear transform to enhance color image while preserving hue attributes and scaling saturation for each color pixel. The algorithm has two features: 1) optimal piecewise affine transform is used for equalizing intensity histogram and the partition of accumulative histogram is optimized using a line regression; 2) the algorithm solves out-of-gamut problem since saturation of each pixel is constrained by scaling inside the boundary of $L C C$ color space in each hue direction. Many experiments have been conducted and the results show that the proposed algorithm enhances color image contrast well while preserving hues better.
\end{abstract}

Keywords: Color image contrast enhancement; Hue-preserving; Histogram equalization; Optimal Adaptive piecewise affine equalization.

\section{Introduction}

Color image contrast enhancement is very important for visual effect from traditional camera to portable device integrated with a compact camera. The enhancement improves quality of image so that the enhanced image is better than the original image. There has increased needs in the fields such as image processing for improving interpretability for visual perception of human being and computer vision for low level vision application in vision system $[1,2]$.

Contrast enhancement transforms pixel values in a given image to new values so that the transformed new image becomes visual pleasant and easy for analysis. There are many methods for image enhancement among which contrast stretching is a simple method while histogram equalization perhaps is the most popular technique [3] for gray scale image. However, histogram equalization (HE) for a single channel cannot be simply generalized to color image since color image contains more channels and the hue component should be processed carefully during contrast enhancement and the over-saturation should be avoided.

The structure of this paper is organized as follows. Section 2 reviews the existing techniques for color image enhancement. Section 3 describes color space conversion in our proposed method. Section 4 explains the proposed optimal piecewise affine equalization. Hue-preserving transform is described in Section 5. In Section 6, experimental results evaluation are reported to demonstrate the performance of the proposed method. Finally, our conclusions are presented in Section 7.

\section{Related Work}

Varieties of methods for color image contrast enhancement have been proposed using histogram equalization technique. Naik [2] reported a hue-preserving color image enhancement algorithm without gamut problem, that is, the non-linear transformed pixel values 
are remained in the valid range of $R G B$ space. The idea is to generalize the common gray scale contrast enhancement method to color image. The transformation of pixel values is proceeded by considering $C M Y$ color space to keep them within the range of $R G B$ space to solve gamut problem while keeping hues unchanged. However, the saturation of the transformed new image becomes low, the result image may look dull and grayish.

There are also several methods such as retinex based adaptive filters [4, 5] using retinex computational model; adaptive color image enhancement based on geometric mean filter, which uses saturation feedback from saturation components and incorporates spatial information into luminance [6]. Local contrast enhancement of images using greedy algorithm is also proposed [7], which uses an optimization technique to achieve local contrast enhancement by estimating the average local contrast. To keep the hue component unchanged during contrast enhancement, a method was proposed which enhances color image by using an exact HSI color model [8].

Recently, a color and contrast enhancement method called piecewise affine equalization (PAE) has been reported [9] . PAE combines a piecewise affine transform of pixel intensity value and histogram equalization. The idea of PAE is very simple which performs a piecewise linear transform of the pixel intensity values such that the new cumulative distribution function will become approximately linear, while the slope of the contrast variation is locally controlled. The PAE can avoid brutal noise enhancement as the authors claimed. However, the output range is partitioned into equal intervals, which may squeeze pixels with sharp change into a narrow output range and then compress histogram even it originally has large contrast. Moreover, PAE is not a hue-preserving algorithm since it processes each color channel independently or only processes intensity component with the restriction that ratios among $R, G$, and $B$ are preserved.

Motivated by piecewise affine equalization approach, the algorithm proposed in this paper using an optimal piecewise affine histogram equalization while keeping hues unchanged for all pixels. The partition of output range is optimized using a line clustering algorithm to make each partition fit a line with minimum residues. This consideration will avoid squeezing pixels with sharp change into a narrow output range. Moreover, to preserve hue for every pixel, pixels in $R G B$ color space are transformed to $L C C$ color space [10]. This is an invertible and simple transform. To solve out-of-

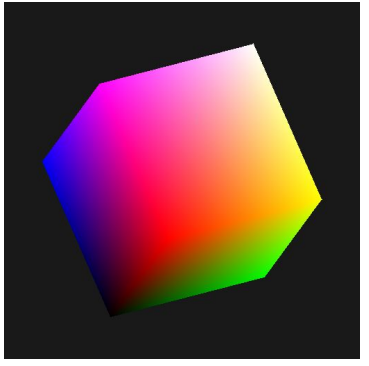

(a)

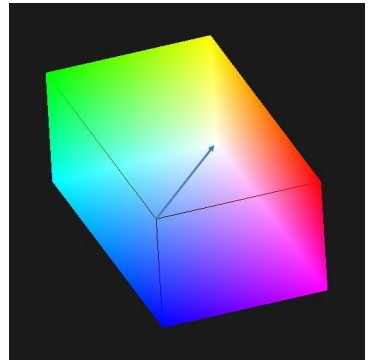

(b)
Figure 1 Color spaces: (a) $R G B$ cube; (b) $L C C$ parallelepiped shape.

gamut problem while preserving hues, saturations are scaled on chromatic plane according to the pixel's intensities before and after histogram equalization.

\section{Color Space Conversion}

The proposed optimal piecewise affine histogram equalization (abbreviated as OPAE) is applied to the intensity component which is separated from the given $R G B$ values. The proposed algorithm first enhances the intensity components and then scales saturations in the original hue direction on chromatic planes to preserve hue components. Since the commonly used $R G B$ (Fig.1(a)) space is not very intuitive for color perception, it has no direct luminance and chrominance information. An intermediate color space is required for color image enhancement. This color space should contain luminance and chrominance separately. Many common used color spaces such as $H S V$ (or $H S I)$ and $C I E L a b$ may be selected as an intermediate color space, which are intuitive but computationally complex. We use a simple and intuitive luminance chrominance color space $L C C$ (Fig.1(b)) as an intemediate space to accomplish color image enhancement. Given an color image in $R G B$ space, it is linearly converted into a different representation in $L C_{1} C_{2}$ using equation

$$
\left(\begin{array}{c}
L \\
C_{1} \\
C_{2}
\end{array}\right)=\left(\begin{array}{rrr}
0.2990 & 0.5870 & 0.1140 \\
0.5000 & 0.5000 & -1.0000 \\
0.8660 & -0.8660 & 0.0000
\end{array}\right)\left(\begin{array}{l}
R \\
G \\
B
\end{array}\right),
$$

where $R, G$, and $B$ represent values for each channel. While $L$ is used for luminance computed from the linear combination of $R, G$, and $B$ as the same with most of color space conversion, the transformed $C_{1}$ and $C_{2}$ represent chromatic information of Yellow-Blue and Magenta-Red/Cyan-Green respectively as shown in Fig.2. Suppose that $R, G$ and $B$ are in range $[0,1]$, chroma $C 1$ is in range $[-1.0,1.0]$ while chroma $C 2$ is 


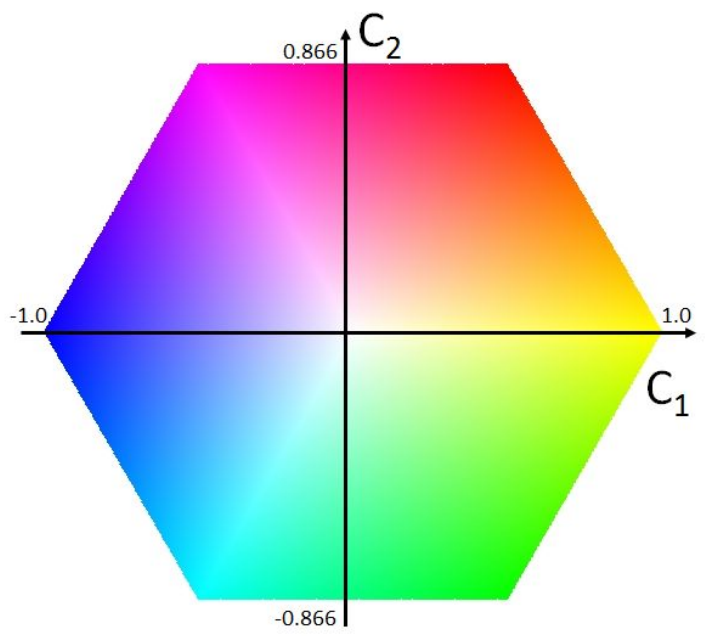

Figure 2 Visualization of the gamut of $L C C$ color spaces viewed along luminance axis from white to black. The chroma plane has two perpendicular axes of Yellow-Blue and Magenta-Red/Cyan-Green.

in range $[-0.866,0.866]$. The typical color Red, Yellow, Green, Cyan, Blue, and Magenta are well spaced on the "Hue circle". Like $H S V$ color space, this is an invertible transform between $R G B$ and $L C C$ color spaces using equation (1) and its inverse transform. Fig.1 shows these color spaces. Fig.1(a) is the well-known $R G B$ cube and Fig.1(b) is a $L C C$ parallelepiped shape transformed from $R G B$ cube using equation (1) in which the arrow means intensity $L$ axis.

\section{Optimal Piecewise Affine Equalization}

After color space conversion, an intermediate color space $L C C$ is constructed. The luminance channel represents a gray scale image. Suppose $L(i)$ be a given gray scale image with $M$ pixels, where $L(i)$ stands for pixel value at $i$. Pixel intensity value is in the range $[0,255]$ and positions are $i=0,1,2, \cdots, M-1$. The cumulative histogram function is notated as $H(x)$ : $[0,255] \rightarrow[0,1]$ where $x$ is a gray level.

Histogram equalization performs a mapping from an input intensity $x$ to an output intensity $y$. Suppose that the output range $[0,255]$ is equally partitioned into $N$ segments with $0=y_{0}<y_{1}<y_{2}<\cdots<$ $y_{N}=255$. Since all segments are equal in length, the partition point is expressed as $y_{k}=255 k / N$ for $k=0,1,2, \cdots, N$.

With this partition in the output range, the corresponding partition in the input range can be defined as $x_{k}=H^{-1}\left(y_{k} / 255\right)$ for $k=0,1,2, \cdots, N$. Since $H(x)$ is a cumulative histogram, it is a non-decrease function, $H^{-1}(z)$ is computed as the minimum value at which $H(x) \geq z$.

For the $k$-th segment $\left[x_{k}, x_{k+1}\right]$ in the input range, a linear transform $T_{k}(x)$ is constructed so that $T_{k}(x)$ maps $\left[x_{k}, x_{k+1}\right]$ into a segment $\left[y_{k}, y_{k+1}\right]$ in the output range. The mapping is represented as $T_{k}(x)=$ $y_{k}+s_{k}\left(x-x_{k}\right)$, where $k=0,1,2, \cdots, N-1$ and $x \in\left[x_{k}, x_{k+1}\right]$. The slope can be computed as $s_{k}=$ $\left(y_{k+1}-y_{k}\right) /\left(x_{k+1}-x_{k}\right)$. It is known that smaller slope compresses histogram thus using smaller slope will be at risk of losing much contrast and larger slope stretches histogram thus noise amplification will be occurred especially in dark areas.

However, the number of pixels for intensity levels in some input range $\left[x_{k}, x_{k+1}\right]$ may have dramatic change, the curve in these range cannot be approximated using a simple line equation $y=s x+t$. Since a linear transform is simple, using a polynomial over order 2 is not a good idea. To solve this problem, the residues could be reduced by adjusting the joint point between segments to make linear model fit the segments with least errors. This is regarded as an optimization problem modeled as cost function

$$
E=\sum_{k=0}^{N-1} \sum_{j=0}^{p^{k}-1}\left(s_{k} x_{j}^{k}+t_{k}-y_{j}^{k}\right)^{2},
$$

where $p^{k}$ is the number of intensity levels in the $k$ th segment, $s_{k}$ and $t_{k}$ are slope and intercept of line equation. $x_{j}^{k}$ and $y_{j}^{k}$ are $j$-th intensity levels in the input range and output range respectively for the $k$-th segment.

Since $p^{k}$ is unknown, it is difficult to find an analytic solution of the above optimization problem. Fortunately, this problem is essentially a clustering problem. It equals to divide cumulative histogram into several segments such that the points in each segment lie on a line as could as possible. Algorithmically, it can be solved by adjusting segment joint points $x_{k}$ and $x_{k+1}$ to fit a line model to the data from $y_{k}$ to $y_{k+1}$ so that the fitting errors are minimized. Note that the beginning point $x_{0}$ and the ending point $x_{N}$ are kept unchanged.

The slope of linear transform should not be too small or too large, it is constrained as

$$
s_{k}=\left\{\begin{array}{ll}
\max \left(s_{k}, s_{\min }\right) & s_{k}<1 \\
\min \left(s_{k}, s_{\max }\right) & s_{k} \geq 1
\end{array} .\right.
$$

By default the parameters $s_{\min }$ and $s_{\max }$ are set to 0.0 and 3.0 respectively. These parameters affect ap- 


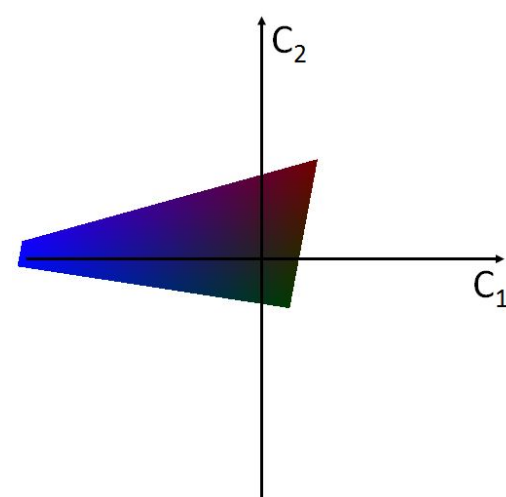

(a)

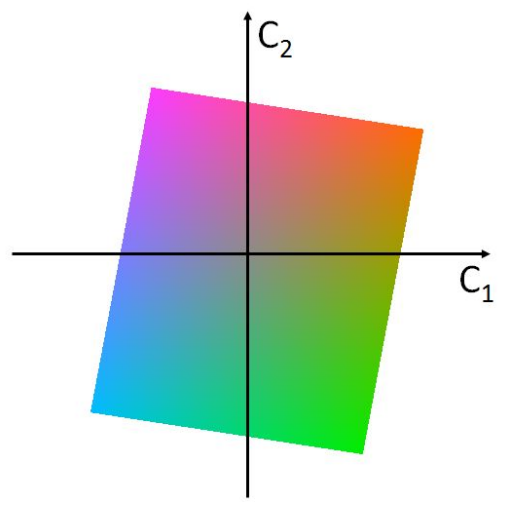

(d)

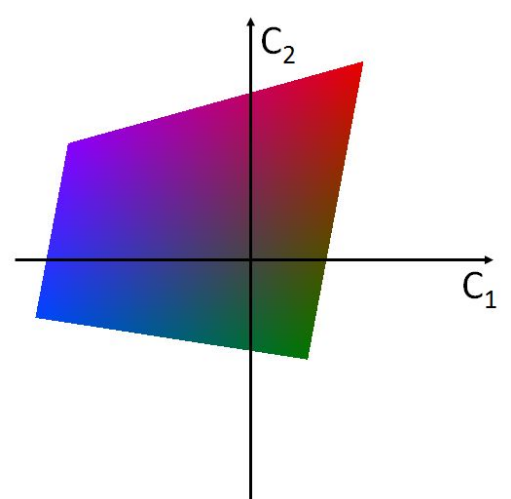

(b)

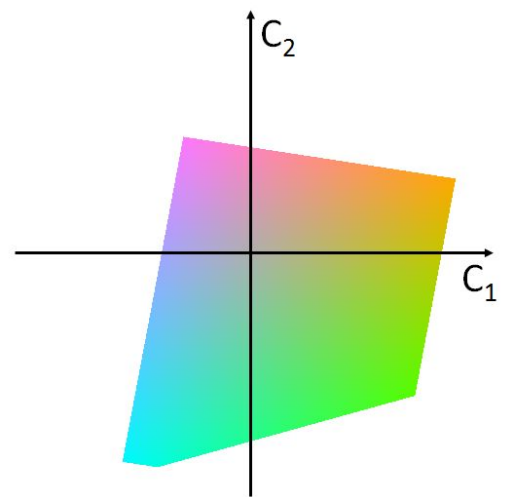

(e)

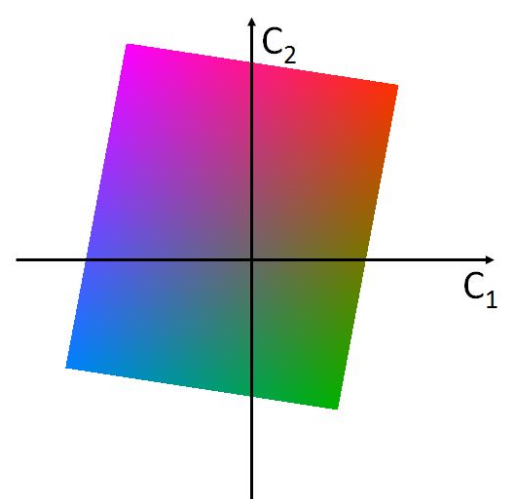

(c)

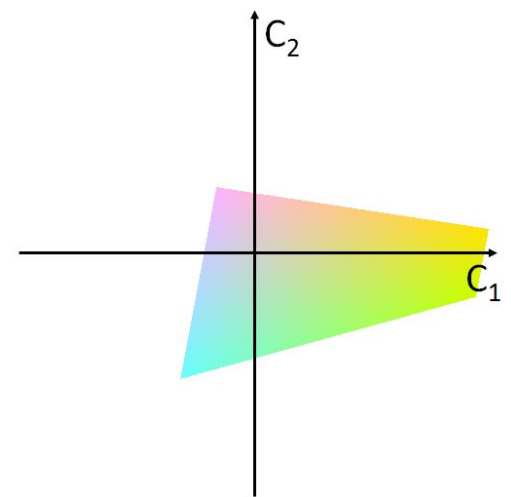

(f)

Figure 3 Chromatic cross section examples in $L C C$ color space: (a) $L=35$; (b) $L=70$; (c) $L=105$; (d) $L=140$; (e) $L=175$; (f) $L=210$.

pearance of result image. However, the default values produce better results for most of images as reported in reference [9].

\section{Hue-Preserving Transform}

For color image enhancement, it is not enough by only applying histogram equalization to the luminance component. The hue for each pixel should be preserved and the saturation should be adjusted so that the final pixel values are inside a $R G B$ cube, that is, in the color gamut of $R G B$ color space.

\subsection{Luminance contrast enhancement}

Hue component should be preserved during color image contrast enhancement. To preserve hues for all pixels in the image, hues should be computed for all pixels. However, $R G B$ color space is unsuitable for computing hues of pixels. A different color space owning a luminance component may be convenient for the computation. Pixels are all converted into $L C C$ color space with three channels: one luminance channel and two chromatic channels. The contrast enhancement mentioned in the last section is applied to the luminance channel. If the new luminance value is $L^{\prime}$ after enhancing the luminance component $L$, the new chromatic components $C_{1}^{\prime}$ and $C_{2}^{\prime}$ are computed while the hues should be preserved. The computation of chromatic components should guarantee that the pixel values converted from $L^{\prime} C_{1}^{\prime} C_{2}^{\prime}$ color space are inside the color gamut of $R G B$ color space.

\subsection{Cross section of $L C C$ color space}

Suppose the size of $R G B$ cube be $255^{3}$ with $R, G$, and $B$ being in the range $[0,255]$. When this cube is transformed using equation (1), the result is a parallelepiped shape as shown in Figure 1(b) which forms an $L C C$ color space with $(0,0,0)$ as origin and the diagonal line from the origin as $L$ axis ranging from 0 to 255 as the arrow shows. The chromatic axes $C_{1}$ and $C_{2}$ are shown in Figure 2 and Figure 3.Planes perpendicular to the $L$ axis are chrominance planes which crossed with $L$ axis. Although the gamut of $L C C$ color space is a regular parallelepiped shape, when viewed along the luminance axis from white to black as shown in Figure 2, the cross sections have very different shapes for different intensities. Figure 3 shows six cross sections for different intensities as examples. Specially, 
when $L=0$ and $L=255$ the shapes degenerate to a single point. With different $L$ value, the cross section may be a triangle, a quadrangle, and a pentagonshaped.

\subsection{Hue preserving and saturation scaling}

When the luminance component $L$ of a given pixel $\left(L, C_{1}, C_{2}\right)$ in $L C C$ space is linearly transformed to $L^{\prime}$ in the process of optimal piecewise affine histogram equalization, the pixel $\left(L^{\prime}, C_{1}^{\prime}, C_{2}^{\prime}\right)$ is calculated to preserve hue component. Cross sections are denoted as $P$ and $P^{\prime}$ for $L$ and $L^{\prime}$.

Since the shape of $L C C$ color space is not a cylinder, the chroma of the original pixel with luminance $L$ cannot be used as the chroma of the pixel with luminance $L^{\prime}$ after enhancement. The reason is that if the original chroma is used, some pixels will be out of the gamut of $R G B$ color space. To solve this problem, a solution is to clamp pixel values enforce them be inside the gamut. The clamping is to take pixels that are outside $L C C$ gamut boundary and to project them onto the boundary of the parallelepiped. However, this approach may result in over-saturated for some pixels.

In practice, instead of using clamping technique, a scaling scheme works well. The scaling scheme uses the maximum saturations of pixels before and after contrast enhancement. The maximum saturation $S_{\max }$ of a pixel is computed in the hue direction of $\left(C_{1}, C_{2}\right)$ on the cross section $P$. The maximum saturation $S_{\max }$ is in fact the distance from the luminance axis to the boundary in the hue direction. Since the shapes of cross sections are irregular, it is hard to compute the maximum saturation in an analytic way. A heuristic algorithm is used to search the maximum saturation under the condition that the point in $L C C$ parallelepiped shape should also be inside the $R G B$ cube when transformed back. If a ray is emanated from $(L, 0,0)$ in the hue direction and hit the boundary, the maximum saturation is computed.

The saturation of $\left(L, C_{1}, C_{2}\right)$ is the distance $D$ of this pixel from the axis $L$ in $L C C$ color space. The saturation of this pixel should be computed when the luminance becomes $L^{\prime}$ from $L$. Since the hue component should be preserved, $\left(C_{1}^{\prime}, C_{2}^{\prime}\right)$ on cross section $P^{\prime}$ has the same direction with $\left(C_{1}, C_{2}\right)$ on the cross section $P$. The maximum saturation $S_{\max }^{\prime}$ in the direction $\left(C_{1}^{\prime}, C_{2}^{\prime}\right)$ on cross section $P^{\prime}$ is also searched in a heuristic way. The distance $D^{\prime}$ from the new pixel to the $L$ axis is computed by scaling as

$$
D^{\prime}=\frac{D * S_{\max }^{\prime}}{S_{\max }}
$$

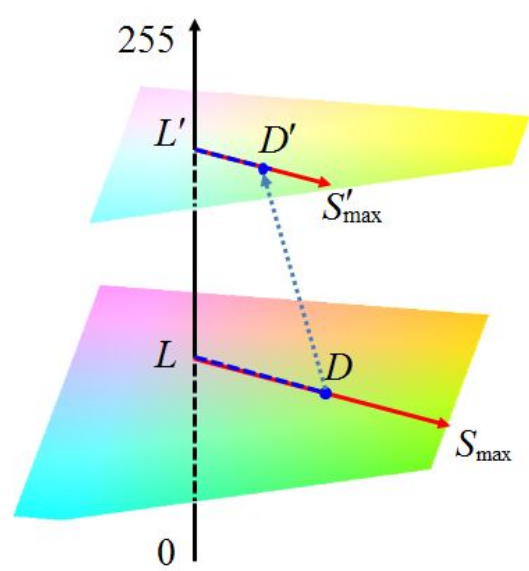

Figure 4 Illustration of hue preserving and saturation scaling with $L=175$ and $L^{\prime}=210$ as an example.

Figure 4 illustrates the above process. For example, if the luminance of a pixel is $L$ and is enhanced to $L^{\prime}$. The cross sections have different shapes for these intensities. The hues should be unchanged, the maximum saturations from those hue directions are found on the boundary. The original saturation is $D$, the new saturation $D^{\prime}$ is easily calculated using expression (4).

The computed distance $D^{\prime}$ is in fact the saturation of pixel $\left(L^{\prime}, C_{1}^{\prime}, C_{2}^{\prime}\right)$. This process preserves hue while adjust saturation by scaling. After all pixels are transformed in this way, the resulted pixels in $L C C$ color space are transformed back into $R G B$ color space.

\section{Experimental Results and Comparisons}

To confirm the validity of the proposed method, many experiments have been conducted. For comparisons, parameters $N, s_{\min }, s_{\max }$ are set to be 5, 0, and 3, respectively in all experiments. Figure 5 (a) shows an original color image with size of $513 \times 385$ and Figure 5 (b) is a result image by running the original piecewise affine histogram equalization (PAE). Although the contrast is enhanced, the hues of some pixels are not preserved. Figure 5 (d) is a result produced by the proposed algorithm. By observing and comparing Figure 5(d) with Figure 5(a), one can know that the image is enhanced and the hues of pixels in Figure 5(d) are well preserved with high saturation. For comparison, Figure 5(c) is an enhanced image by using general histogram equalization, hues of pixels are changed and the white pixels are heavily over-saturated.

Another experimental result is illustrated in Figure 6. Figure 6(a) is an original image with size of $768 \times$ 512 and Figure 6(b) is produced by applying PAE to each color channel. While contrast is enhanced, the hues seem preserved well in this case. However, part 


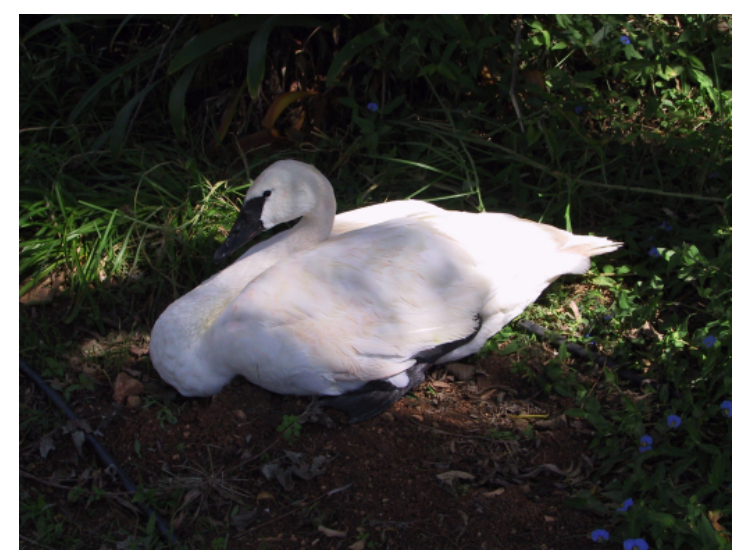

(a)

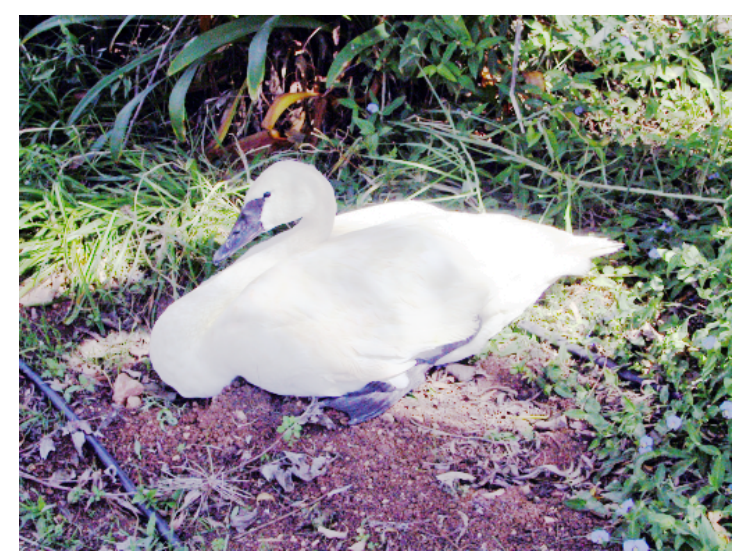

(c)

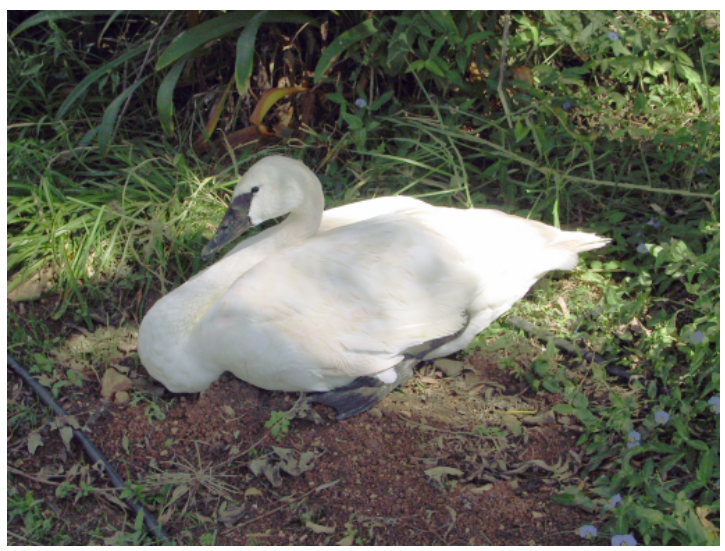

(b)

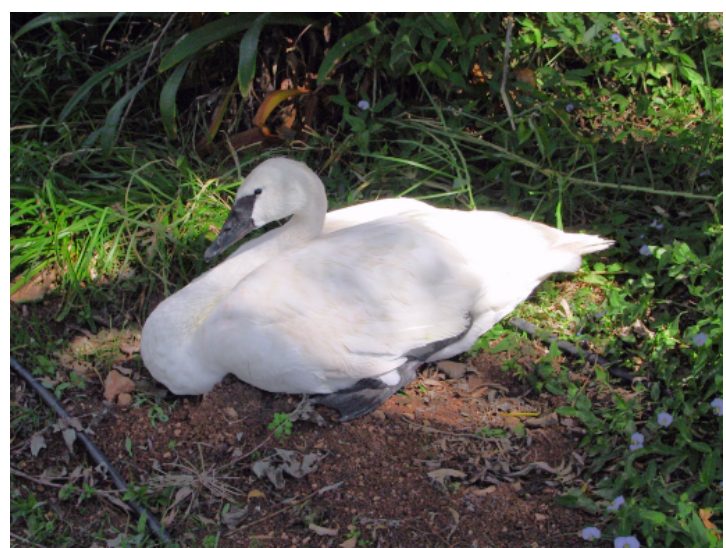

(d)

Figure 5 Experimental result: (a) original color image; (b) result produced by using piecewise affine equalization(PAE), hues of some pixels are changed; (c) result using general histogram equalization, hues are changed and the white pixels are over-saturated; (d) result using our proposed hue-preserving optimal piecewise affine equalization.

of sweater on the shoulder is heavily over-saturated. Figure 6 (c) is also produced by applying PAE to the intensity channel while maintaining the ratios of $R$, $G$, and $B$. It is observed that the hues of most of pixels are severely changed in this image. Pseudo edges are produced on the background near girl shoulder due to the equal partition of output range, that is, almost similar pixels are transformed using different slopes. Figure 6(d) is a result delivered by the proposed method. It is obviously that the contrast is enhanced and the hues are well preserved although the saturation of some pixels is reduced due to solving out of gamut problem.

Figure 7 illustrates a different experiment. The original image with size $768 \times 512$ is shown in Figure 7(a) which is colorful and has relatively high contrast. When applied PAE to each color channel, the contrast is enhanced but the result image looks blueish as shown in Figure 7(b). If the PAE is applied to the intensity component while maintaining the ratios among
$R, G$ and $B$, the result image is Figure 7(c). It seems that the hues are well preserved, however, too many pixels on hats especially on the yellow hat are obviously over-saturated. Figure 7(d) is the result by using the proposed approach. One can observe that this color enhanced image is much better than Figure 7(b) and Figure 7(c).

Parameters are designed for quantitatively evaluating algorithms. These parameters are contrast enhancement performance $C=\left(S_{\text {out }}-S_{\text {in }}\right) / S_{\text {in }}$, luminance enhanced performance $L=\left(L_{\text {out }}-L_{\text {in }}\right) / L_{\text {in }}$. $S_{\text {out }}$ and $S_{i n}$ are variances of luminance of output and input image. $L_{\text {out }}$ and $L_{\text {in }}$ are means of luminance of output and input image. A mean squared error $(M S E)$ of hues between the original image and the enhanced

Table 1 Performance comparison.

\begin{tabular}{llll}
\hline Parameter & HE & PAE & Ours \\
\hline Contrast & 0.1520 & 0.1302 & 0.1837 \\
\hline Luminance & 1.4436 & 0.9759 & 0.8960 \\
\hline Hue variation & $0.0964^{\circ}$ & $0.0902^{\circ}$ & $0.0100^{\circ}$ \\
\hline
\end{tabular}




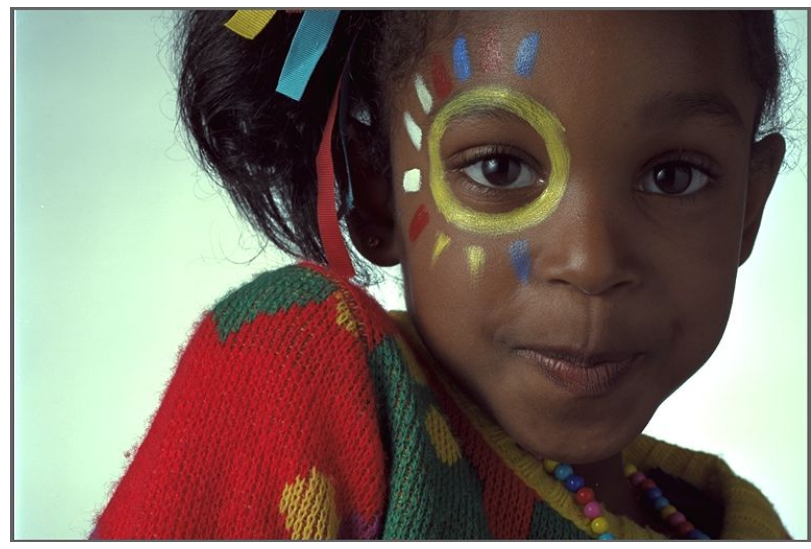

(a)

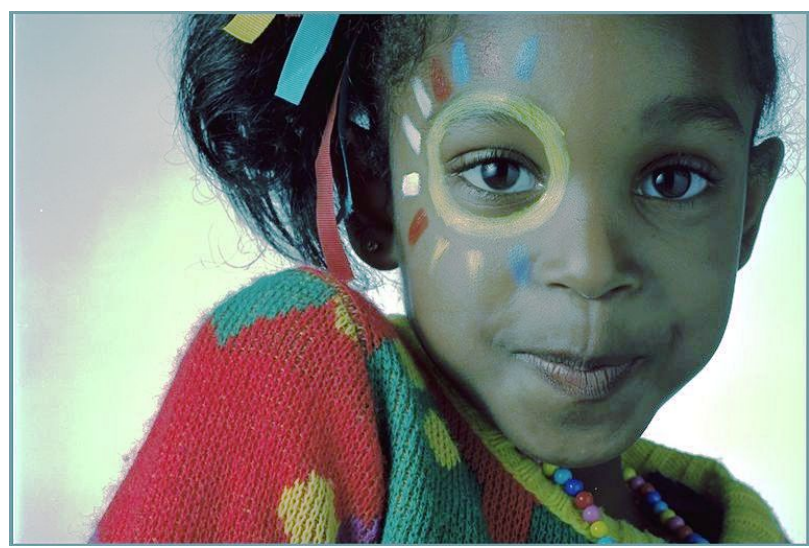

(c)

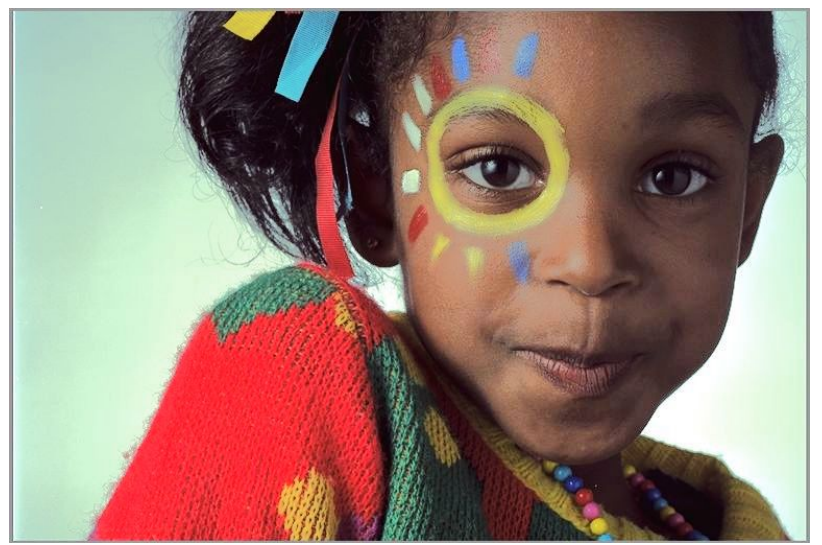

(b)

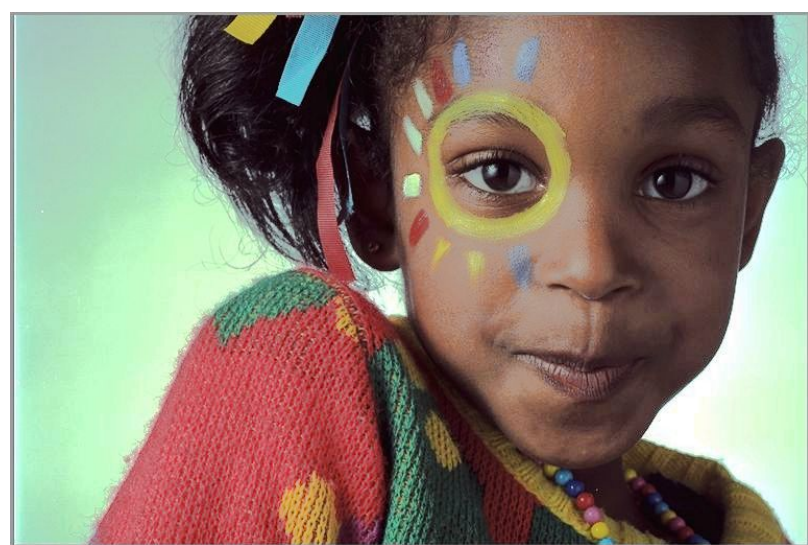

(d)

Figure 6 Experimental result: (a) original color image; (b) result produced by using piecewise affine equalization(PAE) to each channels, the red sweater on the shoulder is heavily saturated; (c) result produced by using piecewise affine equalization(PAE) to intensity components and then compute RGB from the intensity with restriction that the ratios among $R$, $G$, $B$ are preserved, the hues for most of pixels are severely changed; (d) result produced by using optimal piecewise affine histogram equalization to intensity component in LCC color space, scaled saturation is computed while preserving hues for each pixel.

image is computed for examining the variation of hues before and after enhancement. As an instance, images in Figure 5 are used for computing values of the above parameters. The performance comparison is shown in Table 1. The first column is the evaluation parameters; the second and third columns show values of parameters when using HE and PAE methods; the last column is for the proposed algorithm. It is clear that the proposed method is the best one by observing these values. The contrast performance exceed the other two's. Especially, the hue variation of ours is almost zero degree, that is, the proposed method is hue-preserving.

\section{Conclusion}

A new approach for color image enhancement was proposed. For histogram equalization, the output range of pixels is partitioned in an optimal way to reduce the residues for each partition when fitting to a linear transform model.Equalizing histogram in this way avoids squeezing pixels with sharp change into a narrow output range. Given an input image, all pixels are converted to $L C C$ color space from $R G B$ space to separate luminance component and chromatic components. After the luminance component is enhanced using the proposed method, the saturations of all pixels are computed by scaling on a different cross section plane while preserving hues. This hue-preserving and saturation scaling can solve out-of-gamut problem. The experiments demonstrate that the results are visually better than the original piecewise affine histogram equalization.

Although the proposed method achieved good performance, there still are several limitations. During constructing piecewise affine functions, it is better to 


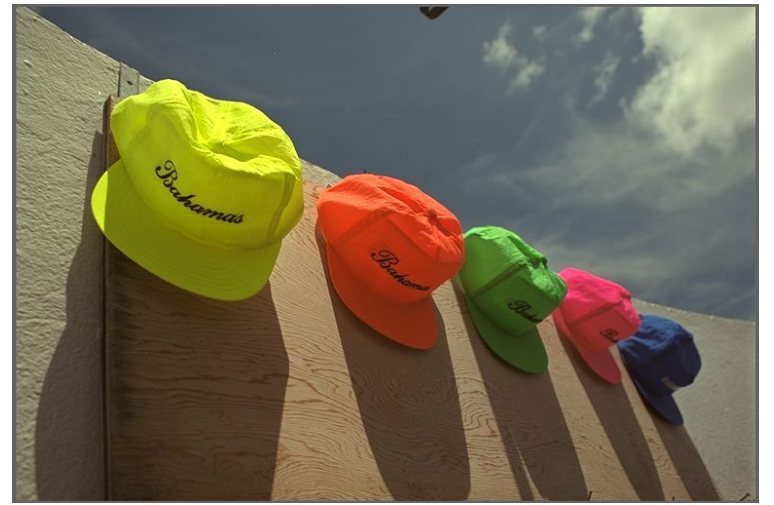

(a)

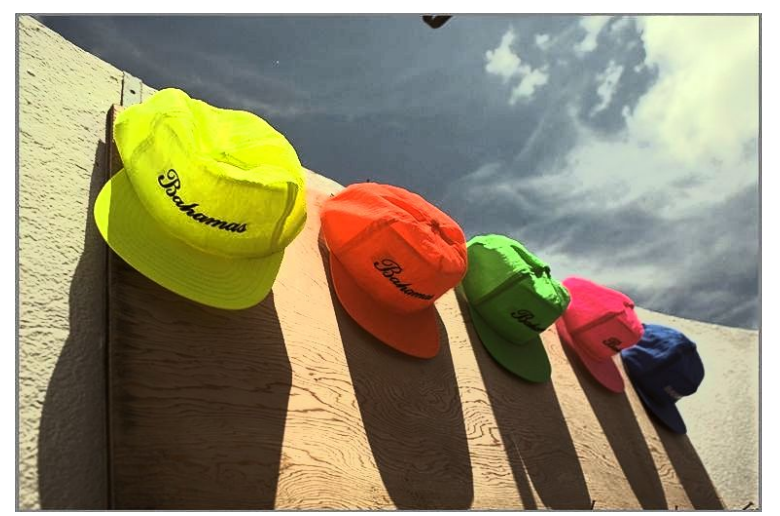

(c)

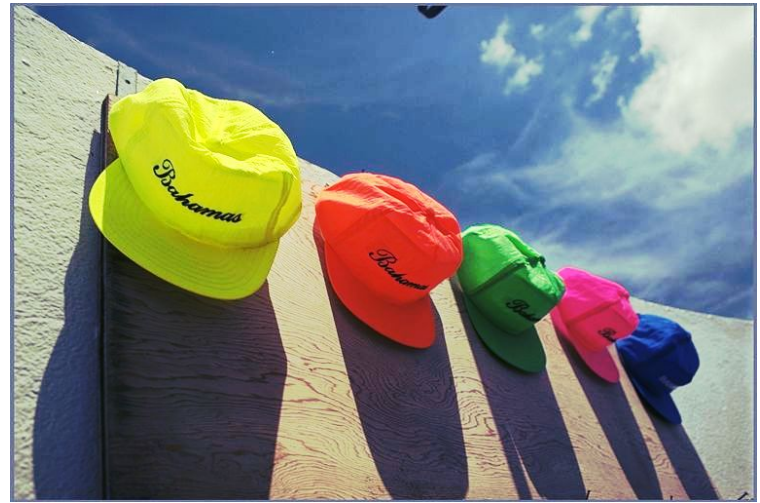

(b)

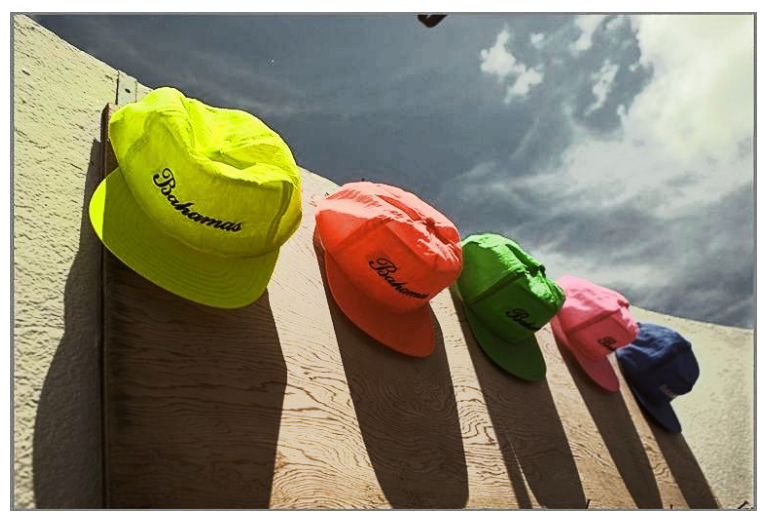

(d)

Figure 7 Experimental result: (a) original color image with relatively high contrast; (b) result produced by using piecewise affine equalization(PAE) to each channels, the image appears blueish and some pixels are over-saturated, especially on the yellow hat; (c) result produced by using piecewise affine equalization(PAE) to intensity components and then compute RGB from the intensity with restriction that the ratios among $R, G$, and $B$ are preserved. The hues seem preserved, however, too many pixels are over-saturated on these hats; (d) result produced by the proposed method. The optimal piecewise affine histogram equalization is applied to the intensity component in $L C C$ color space, scaled saturation is computed while preserving hues for all pixels.

decide automatically the number of segments and the parameters $s_{\min }$ and $s_{\max }$ using image statistics. For some pixels such as on the shoulder as shown in Figure $6(d)$, the saturation become weak compared with the original one. This may be improved by using other method such as saturation equalization. Combining retinex theory with the proposed hue-preserving transform may be another choice for color image enhancement. The relation among luminance, hue and saturation would be furthermore investigated and more natural enhancement technique should be considered in future work.

\section{Acknowledgment}

This work is a part of the research supported by the Chunhui project of the Education Ministry of China under Grants No.Z2012100 and Z2014020, and the National Nature Science Foundation of China under
Grant No.61462072. We are particular grateful to reviewers who gave us detailed precious comments for improving this paper.

\section{References}

[1] D. Menotti, L. Najman, J. Facon, A. de A. Albuquerque, "Fast Hue-Preserving Histogram Equalization Methods for Color Image Contrast Enhancement", International Journal of Computer Science \& Information Technology (IJCSIT), Vol. 4, No. 5, pp.243-257, 2012.

[2] S. K. Naik, C. A. Murthy, "Hue-Preserving Color Image Enhancement without Gamut Problem", IEEE Transaction on Image Processing, Vol. 12, No. 12, 2003.

[3] R. C. Gonzalez, R. E. Woods, "Digital Image Processing", Third Edition, Pearson International Edition, Prentice Hall, pp.129-166, 2010. 
[4] L. Meylan, S. Susstrunk, "Color Image Enhancement using a Retinex-based adaptive Filter", Proceedings of IS \& T Second European Conference on Color in Graphics, Image, and Vision (CGIV 2004), Vol. 2, pp.359-363, 2004.

[5] S. Chen, A. Beghdadi, "Natural Enhancement of Color Image", EURASIP Journal on Image and Video Processing, Vol. 2010, ID:175203, 2010.

[6] M. C Hanumantharaju, M. Ravishankar, D. R. Rameshbabu, "Adaptive Color Image Enhancement Based on Geometric Mean Filter", Proceedings of the 2011 International Conference on Communication, Computing \& Security, pp. 403-408, 2011.

[7] K. Subr, A.Majumder, S. Irani, "Greedy Algorithm for Local Contrast Enhancement of Images", Image Analysis and Processing - ICIAP 2005, Lecture Notes in Computer Science, Vol. 3617, pp.171-179, 2005.

[8] C. L. Chen, D. C. Tseng, "Color Image Enhancement with Exact HSI color Model", International Journal of Innovative Computing, Information and Control, Vol. 7, No. 12, pp.6691-6710, 2011.

[9] J. L. Lisani, A. B. Petro, C. Sbert, "Color and Contrast Enhancement by Controlled Piecewise Affine Histogram Equalization", Image Processing On Line (IPOL)", No.2, pp.243-265, 2012.

[10] M. Bratkova, S. Boulos, P. Shirley, "oRGB: A Practical Opponent Color Space for Computer Graphics", Computer Graphics \& Applications, Vol. 29, Issue 1, 2009. 